\title{
On the Drazin index of regular elements
}

\author{
Pedro Patrício ${ }^{a *}$ A. Veloso da Costa ${ }^{a}$
}

November 29, 2008

\begin{abstract}
${ }^{a}$ Centro de Matemática, Universidade do Minho, Campus de Gualtar, 4710-057 Braga, Portugal; e-mail:\{pedro,aveloso\}@math.uminho.pt

Research supported by CMAT - Centro de Matemática da Universidade do Minho, Portugal, and by the Portuguese Foundation for Science and Technology - FCT through the research program POCTI.
\end{abstract}

Keywords: Drazin inverse, Drazin index, Dedekind finite ring, regular ring

AMS classification: 15A09 (primary), 16A14 and 16A30 (secondary)

\begin{abstract}
It is known that the existence of the group inverse $a^{\#}$ of a ring element $a$ is equivalent to the invertibility of $a^{2} a^{-}+1-a a^{-}$, independently of the choice of the von Neumann inverse $a^{-}$of $a$. In this paper, we relate the Drazin index of $a$ with the Drazin index of $a^{2} a^{-}+1-a a^{-}$. We give an alternative characterization when considering matrices over an algebraically closed field. We close with some questions and remarks.
\end{abstract}

\section{Introduction}

Let $R$ denote a ring with unity 1 . We say $a \in R$ is regular provided $a \in a R a$. We shall also denote $a\{1\}=\{x \in R \mid a x a=a\}$, whose elements are called von Neumann inverses of $a$. As usual, $a^{-}$is an element of $a\{1\}$. If some power of $a$ is regular then $a$ is said to be weak-regular. As an example, $2 \in \mathbb{Z}_{8}$ is not regular and still it is weak-regular.

In this paper, we will consider Drazin invertibility ([3]) on general associative rings with unity 1 . An element $a$ is said to be Drazin invertible provided there is a common solution to the equations

$$
a^{k} x a=a^{k}, x a x=x, a x=x a,
$$

for some $k \geq 0$. It is well known the uniqueness of the solution, if it exists. As usual, it will be denoted by $a^{D}$. The smallest $k$ for which the equations have a common solution is called the Drazin index of $a$, and denoted by $i(a)$. Two special cases deserve our attention: when $i(a)=0$ means $a$ is a unit, and when $i(a) \leq 1$ defines the so called group invertible

\footnotetext{
${ }^{*}$ Corresponding author
} 
elements. In the former case, the Drazin inverse will be denoted by $a^{\#}$. That is to say, group invertibility is a special case of Drazin invertibility. However, it can be proved that $a$ has a Drazin inverse provided it has a power which is group invertible. Furthermore, the smallest $k$ for which $\left(a^{k}\right)^{\#}$ exists equals the Drazin index $i(a)$ of $a$, and $a^{D}=a^{k-1}\left(a^{k}\right)^{\#}=\left(a^{k}\right)^{\#} a^{k-1}$.

We will make use of left and right ideals generated by a power of $a$. In fact, $i(a)=k$ if and only if $k$ is the smallest for which $a^{k} R=a^{k+1} R$ and $R a^{k}=R a^{k+1}$, or equivalently, $a^{k} \in a^{k+1} R \cap R a^{k+1}$. This implies, for any $n \geq k$, the relation $a^{n} \in a^{n+1} R \cap R a^{n+1}$. As a special case, $a^{\#}$ exists if and only if $a \in a^{2} R \cap R a^{2}$ if and only if $a R=a^{2} R, R a=R a^{2}$. The left [resp. right] index of $a$ is the smallest value $p$ [resp. $q$ ] for which $a^{p+1} R=a^{p} R$ [resp. $R a^{q+1}=R a^{q}$. It was shown in [3] (cf. [7, page 11]) that if $p$ and $q$ are finite then $p=q=i(a)$.

R. Cline showed in [2] how to relate $(a b)^{D}$ with $(b a)^{D}$, namely $(a b)^{D}=a\left((b a)^{D}\right)^{2} b$. This equality is known as Cline's formula. According to [7, page 16], the indices $i(a b)$ and $i(b a)$ differ at most by unity. That is to say, $|i(a b)-i(b a)| \leq 1$. When considering matrices over a field $\mathbb{F}$, this corresponds to $\psi_{A B}(\lambda)=\lambda^{0, \pm 1} \psi_{B A}(\lambda)$, where $\psi_{A B}$ and $\psi_{B A}$ denote, respectively, the minimal polynomial of $A B$ and $B A$. If, in addition, $\mathbb{F}$ is algebraically closed, then every matrix is similar to a diagonal block matrix with Jordan blocks, known as the Jordan canonical (or normal) form. This gives, in particular, the core-nilpotent decomposition: given a matrix $A$ over $\mathbb{F}$, there are (possibly absent) matrices $U$ invertible and $N$ nilpotent with nilpotency index, say, $k$, for which $A \approx\left[\begin{array}{cc}U & 0 \\ 0 & N\end{array}\right]$, where $\approx$ denotes matrix similarity. In this case, $A^{D} \approx$ $\left[\begin{array}{cc}U^{-1} & 0 \\ 0 & 0\end{array}\right]$. Note that Drazin invertibility is invariant to matrix similarity, and recall similar matrices have the same minimal polynomial. Therefore, this means $\psi_{A}=l \mathrm{~cm}\left(\psi_{U}, \psi_{N}\right)$. As $U$ is invertible and $N$ is nilpotent with nilpotency index $k$ then $\psi_{U}(0) \neq 0$ and $\psi_{N}(\lambda)=\lambda^{k}$, and hence $\psi_{A}(\lambda)=\lambda^{k} \psi_{U}(\lambda)$. As a conclusion, the Drazin index of $A$ equals the algebraic multiplicity (possibly zero) of 0 as a root of the minimal polynomial $\psi_{A}$ of $A$. With no surprise, the multiplicity of the root 0 (if any) of the minimal polynomial of a matrix over a field is usually called the index of the matrix.

A ring $R$ is said to be Dedekind finite if $x y=1$ implies $y x=1$. An important property of these rings is that, given $e^{2}=e, f^{2}=f \in R$, then, as in [4, Theorem 1], the equivalence of the following hold:

1. $R$ is Dedekind finite;

2. $e R \subseteq f R$ and $e \sim f$ imply $e R=f R$;

3. $R e \subseteq R f$ and $e \sim f$ imply $R e=R f$;

where $e \sim f$ means $e R \cong f R$ as right $R$-modules, or equivalently, $R e \cong R f$ as left $R$-modules.

As a consequence (cf. [4, Theorem 2]), if $a^{k}$ is regular (that is, $a$ is weak-regular) then the equality $a^{k} R=a^{k+1} R$ is equivalent to the existence of the Drazin inverse of $a$, with $i(a) \leq k$, provided $R$ is Dedekind finite. In this case, the equality $a^{k} R=a^{k+1} R$ implies 
$R a^{k} \cong R a^{k+1}$ as left $R$-modules by taking $\varphi\left(y a^{k}\right)=y a^{k+1}$ as the desired isomorphism. Since trivially $R a^{k+1} \subseteq R a^{k}$ then $R a^{k+1}=R a^{k}$, and therefore $i(a) \leq k$.

If $R$ is not Dedekind finite, then such an outcome cannot be expected. Indeed, if $u v=$ $1 \neq v u$ then $u^{D}$ does not exist and still $u^{\ell} R=R=u^{\ell+1} R$, for any natural $\ell$.

\section{Main results}

The Puystjens-Hartwig Theorem ([10]) characterizes the group invertibility of a regular element in terms of units. We may rewrite it as the equivalences $(1) \Leftrightarrow(2) \Leftrightarrow(4)$ in the proposition below. We add two more simpler equivalences.

Proposition 2.1. Given a regular $a \in R$, the following conditions are equivalent:

1. $i(a) \leq 1$;

2. $i\left(a^{2} a^{-}+1-a a^{-}\right)=0$ for one and hence all choices of $a^{-} \in a\{1\}$;

3. $i\left(a+1-a a^{-}\right)=0$ for one and hence all choices of $a^{-} \in a\{1\}$;

4. $i\left(a^{-} a^{2}+1-a^{-} a\right)=0$ for one and hence all choices of $a^{-} \in a\{1\}$;

5. $i\left(a+1-a^{-} a\right)=0$ for one and hence all choices of $a^{-} \in a\{1\}$.

Proof. Note that $1+a a^{-}(a-1)$ is a unit if and only if $1+(a-1) a a^{-}=a^{2} a^{-}+1-a a^{-}$is a unit, and so $(2) \Leftrightarrow(3)$. The equivalence $(4) \Leftrightarrow(5)$ is obtained similarly.

Recently [13], the existence of the group inverse of a regular element was characterized by means of another unit. We give a proof for the sake of completeness.

Proposition 2.2 (Schmoeger). Given a regular $a \in R$ then $i(a) \leq 1$ if and only if $i\left(1-a a^{-}-\right.$ $\left.a^{-} a\right)=0$, for some $a^{-} \in a\{1\}$.

Proof. Setting $u=1-a a^{-}-a^{-} a$ then obviously $u a=-a^{-} a^{2}$ and $a u=-a^{2} a^{-}$, which lead to $a \in a^{2} R \cap R a^{2}$.

Conversely, taking $a^{-}=a^{\#}$ one can show that $\left(1-a a^{\#}-a a^{\#}\right)^{2}=1$.

Using the same reasoning of the previous result, we may state the following:

Proposition 2.3. Let $a \in R$ be a regular element, and consider the following conditions:

(A) $i(a) \leq 1$.

(B) $i\left(a a^{-}+1-a^{-} a\right)=0$, for some $a^{-} \in a\{1\}$.

(C) $i\left(a^{-} a+1-a a^{-}\right)=0$, for some $a^{-} \in a\{1\}$.

(D) $R$ is Dedekind-finite.

Then 

1. $(A) \Leftrightarrow((B) \wedge(C))$.
2. $(D) \Rightarrow(((B) \vee(C)) \Rightarrow(A))$.

Proof. (1). (A) means $a^{\#}$ exists, and so $(B)$ and $(C)$ both hold by taking $a^{-}=a^{\#}$. Conversely if both $a a^{-}+1-a^{-} a$ and $a^{=} a+1-a a^{=}$are units for some $a^{=}, a^{-} \in a\{1\}$, and since $a\left(a a^{-}+1-a^{-} a\right)=a^{2} a^{-}$and $\left(a^{=} a+1-a a^{=}\right) a=a^{=} a^{2}$, then $a \in a^{2} R \cap R a^{2}$, which in turn means $i(a) \leq 1$.

(2). If $R$ is Dedekind finite, and as in (1), (B) shows $a \in a^{2} R$ and therefore $a \in R a^{2}$ (see [4]), or $(C)$ implies $a \in R a^{2}$ and therefore $a \in a^{2} R$. In either case, $a^{\#}$ exists.

Condition (2) is the best possible, as if $R$ is not Dedekind finite, it is possible to exist a regular $a \in R$ which has no group inverse, and still $a a^{-}+1-a^{-} a$ or $a^{-} a+1-a a^{-}$are units for some $a^{-} \in a\{1\}$. Take $R=\mathcal{B}\left(\ell^{2}\right)$, and the usual orthonormal basis $\left(e_{i}\right)_{i=1}^{\infty}$ in $\ell^{2}$. Define $a \in R$ as $a\left(e_{i}\right)=e_{i+1}$, which is regular and $a^{-}$defined as $a^{-}\left(e_{i}\right)= \begin{cases}e_{i-1} & \text { if } i \geq 2 \\ 0 & \text { otherwise }\end{cases}$ is a von Neumann inverse of $a$. Note $a a^{-} \neq 1=a^{-} a, a a^{-}+1-a^{-} a$ is not a unit and $a^{-} a+1-a a^{-}=2-a a^{-}$is invertible. In fact, $\left(2-a a^{-}\right)^{-1}\left(e_{i}\right)=\left\{\begin{array}{ll}\frac{1}{2} e_{1} & \text { if } i=1 \\ e_{i} & \text { otherwise }\end{array}\right.$.

In the next result, we extend Proposition 2.1.

Theorem 2.4. Let $a \in R$ be a regular non-invertible element. The following conditions are equivalent:

1. $i(a)=k+1$.

2. $i\left(a^{2} a^{-}+1-a a^{-}\right)=k$, for some $a^{-} \in a\{1\}$.

3. $i\left(a^{-} a^{2}+1-a^{-} a\right)=k$, for some $a^{-} \in a\{1\}$.

Proof. $(1) \Leftrightarrow(2)$. When $k=0$ we get Proposition 2.1. So we may consider $k \geq 1$.

Firstly, note that $a^{k+1} a^{-}=\left(a^{2} a^{-}\right)^{k}$, for $k \geq 1$, and secondly $a^{2} a^{-} \in e R e$, where $e=a a^{-}$, from which $\left(a^{2} a^{-}\right)^{D} \in e R e$ with index $k$ if and only if $i\left(a^{2} a^{-}+1-a a^{-}\right)=k$ (see [9]). Alternatively, $x+y$ with $x y=0=y x$ has Drazin index $k$ if and only if $x, y$ have Drazin inverses in which case $k=\max \{i(x), i(y)\}$.

If $i\left(a^{2} a^{-}+1-a a^{-}\right)=k$ then $i\left(a^{2} a^{-}\right)=k$. This means $\left(a^{2} a^{-}\right)^{k+1} R=\left(a^{2} a^{-}\right)^{k} R$ and $R\left(a^{2} a^{-}\right)^{k+1}=R\left(a^{2} a^{-}\right)^{k}$, which in turn gives $a^{k+2} R=a^{k+1} R$ and $R a^{k+2}=R a^{k+1}$. Hence, $i(a) \leq k+1$. Now, if $i(a)=l \leq k$ then $a^{l+1} a^{-} R=a^{l+1} R=a^{l} R=a^{l} a^{-} R$, from which $\left(a^{2} a^{-}\right)^{l} R=\left(a^{2} a^{-}\right)^{l-1} R$, and therefore $k=i\left(a^{2} a^{-}\right) \leq l-1<k$.

Conversely, if $i(a)=k+1$ then $a^{k+2} a^{-} R=a^{k+1} a^{-} R$ and $R a^{k+2} a^{-}=R a^{k+1} a^{-}$, which give $\left(a^{2} a^{-}\right)^{k+1} R=\left(a^{2} a^{-}\right)^{k} R$ and $R\left(a^{2} a^{-}\right)^{k+1}=R\left(a^{2} a^{-}\right)^{k}$. Therefore, $i\left(a^{2} a^{-}\right) \leq k$. Assuming $i\left(a^{2} a^{-}\right)=l<k$ then this would give $a^{l+2} R=\left(a^{2} a^{-}\right)^{l+1} R=\left(a^{2} a^{-}\right)^{l} R=a^{l+1}$ and therefore $i(a) \leq l+1<k+1$. Hence, $i\left(a^{2} a^{-}\right)=k$, which in turn implies $i\left(a^{2} a^{-}+1-a a^{-}\right)=k$.

The equivalence $(1) \Leftrightarrow(3)$ is similar to $(1) \Leftrightarrow(2)$. 
We remark the index of the elements in the Theorem is independent of the choice of the von Neumann inverse of $a$. Therefore, we may state the following result:

Corollary 2.5. Given a regular $a \in R$ and $a^{-} \in a\{1\}$, if $i\left(a^{2} a^{-}+1-a a^{-}\right)=k$ then $i\left(a^{2} a^{=}+1-a a^{=}\right)=k$ for any $a^{=} \in a\{1\}$.

When $k=0$, this gives the known fact that the invertibility of $a^{2} a^{-}+1-a a^{-}$is independent of the choice of $a^{-}$, as in Proposition 2.1.

Lemma 2.6. Given a regular $t \in R$ and a natural $k$,

$$
\left(t+1-t t^{-}\right)^{k}=1+\sum_{i=1}^{k}\left(t^{i}-t^{i} t^{-}\right) .
$$

Proof. The proof is done by induction. The result holds trivially for $k=1$.

Note that $\left(t+1-t t^{-}\right)^{k+1}=\left(t+1-t t^{-}\right)\left(t+1-t t^{-}\right)^{k}$ which equals, by the induction step,

$$
\left(t+1-t t^{-}\right)\left(1+\sum_{i=1}^{k}\left(t^{i}-t^{i} t^{-}\right)\right)
$$

Hence, $\left(t+1-t t^{-}\right)^{k+1}=t+\sum_{i=2}^{k+1}\left(t^{i}-t^{i} t^{-}\right)+1+\sum_{i=1}^{k}\left(t^{i}-t^{i} t^{-}\right)-t t^{-}-\sum_{i=1}^{k}\left(t^{i}-t^{i} t^{-}\right)=$ $1+t-t t^{-}+\sum_{i=2}^{k+1}\left(t^{i}-t^{i} t^{-}\right)=1+\sum_{i=1}^{k+1}\left(t^{i}-t^{i} t^{-}\right)$

Lemma 2.7. Given a regular nilpotent $n \in R$ with $n^{k+1}=0 \neq n^{k}$,

$$
\left(n+1-n n^{-}\right)^{k+1}=\left(n+1-n n^{-}\right)^{k}
$$

Proof. By the previous Lemma, $\left(n+1-n n^{-}\right)^{k+1}=1+\sum_{i=1}^{k+1}\left(n^{i}-n^{i} n^{-}\right)$. Since $n^{k+1}=0$, we have, $\left(n+1-n n^{-}\right)^{k+1}=1+\sum_{i=1}^{k}\left(n^{i}-n^{i} n^{-}\right)=\left(n+1-n n^{-}\right)^{k}$.

Theorem 2.8. Given a regular nilpotent $0 \neq n \in R$ then $n^{k+1}=0 \neq n^{k}$ if and only if $i\left(n+1-n n^{-}\right)=k$, for some $n^{-}$.

Proof. From Lemma 2.7, $i\left(n+1-n n^{-}\right) \leq k$. Note that since the nilpotency index of $n$ is $k+1$ then also $i(n)=k+1$.

We may write $n+1-n n^{-}$as $\left[\begin{array}{ll}1 & n\end{array}\right]\left[\begin{array}{c}1-n n^{-} \\ 1\end{array}\right]$. Using $[2],\left[\begin{array}{ll}1 & n\end{array}\right]\left[\begin{array}{c}1-n n^{-} \\ 1\end{array}\right]$ has a Drazin inverse if and only if $M=\left[\begin{array}{c}1-n n^{-} \\ 1\end{array}\right]\left[\begin{array}{cc}1 & n\end{array}\right]=\left[\begin{array}{cc}1-n n^{-} & 0 \\ 1 & n\end{array}\right]$ has a Drazin inverse, and $\left|i\left(n+1-n n^{-}\right)-i(M)\right| \leq 1$. From [7, Theorem 1], and since $i\left(1-n n^{-}\right)=1$ then $i(n) \leq i(M) \leq i(n)+1$, that is to say, $k+1 \leq i(M) \leq k+2$. Recall $i\left(n+1-n n^{-}\right) \leq k$. 
Now $i(M)=k+1$ implies the possible values for $i\left(n+1-n n^{-}\right)$are $k, k+1, k+2$. If $i(M)=k+2$ then the possible values for $i\left(n+1-n n^{-}\right)$are $k+1, k+2, k+3$. We are left with $i\left(n+1-n n^{-}\right)=k$.

Conversely, suppose $i\left(n+1-n n^{-}\right)=k$ and $i(n)=\ell$, or equivalently, $n^{\ell}=0 \neq n^{\ell-1}$. We want to show $\ell=k+1$. If $\ell \leq k$ then $i\left(n+1-n n^{-}\right) \leq \ell-1<k$ from Lemma 2.7. Therefore $\ell>k$. Now suppose $\ell>k+1$. Setting $M=\left[\begin{array}{c}1-n n^{-} \\ 1\end{array}\right]\left[\begin{array}{cc}1 & n\end{array}\right]=\left[\begin{array}{cc}1-n n^{-} & 0 \\ 1 & n\end{array}\right]$ then $i(M) \in\{k-1, k, k+1\}$ and $\ell=i(n) \leq i(M) \leq i(n)+1=\ell+1$. These inequalities do not hold for the possible values $k-1, k, k+1$ of $i(M)$. Therefore, and since $n$ is nilpotent, $\ell=i(n)=k+1$.

Corollary 2.9. Given a regular nilpotent $0 \neq n \in R, i(n)=k+1$ if and only if $i(n+1-$ $\left.n n^{-}\right)=k$, for some $n^{-}$.

Corollary 2.10. Given a regular nilpotent $0 \neq n \in R$ and $n^{-} \in n\{1\}$ such that $i(n+1-$ $\left.n n^{-}\right)=k$ then $i\left(n+1-n n^{=}\right)=k$, for all $n^{=} \in n\{1\}$.

Theorem 2.11. Let $A$ be a singular square matrix over an algebraically closed field. Then $i(A)=k+1$ if and only if $i\left(A+1-A A^{-}\right)=k$ for some $A^{-}$.

Proof. The case $k=0$ follows from Proposition 2.1. So we may consider $k \geq 1$. For every matrix $A$ there is $C$ invertible and $N$ nilpotent for which $A \approx\left[\begin{array}{cc}C & 0 \\ 0 & N\end{array}\right]$, where $\approx$ denotes matrix similarity. Recall this form is know as the core-nilpotent decomposition. Without loss of generalization, we may consider $A$ to be in its core-nilpotent decomposition. Note that $i(N)=i(A) \geq 2$, and therefore $N \neq 0$. Setting $A^{-}=\left[\begin{array}{cc}C^{-1} & 0 \\ 0 & N^{-}\end{array}\right]$and $U=A+I-A A^{-}$, then $U=\left[\begin{array}{cc}C & 0 \\ 0 & N+I-N N^{-}\end{array}\right]$. Now $i(A)=k+1 \Leftrightarrow i(N)=k+1 \Leftrightarrow i\left(N+I-N N^{-}\right)=$ $k \Leftrightarrow i(U)=k$, which proves the theorem.

\section{Concluding remarks}

We close this paper with some remarks and questions:

1. Cline's formula provides an alternative proof of the main results of [13], as $\mid i(a b)-$ $i(b a) \mid \leq 1$. This implies if $a b$ is a unit then $i(b a) \leq 1$, or equivalently, $(b a)^{\#}$ exists. Also if $\left((a b)^{n}\right)^{\#}$ exists then $i(a b) \leq n$, which implies $i(b a) \leq n+1$, and therefore the existence of $\left((b a)^{n+1}\right)^{\#}$.

2. In this paper, we considered Drazin invertibility of regular elements. Still we must stress that a Drazin invertible element might not be regular. In this paper, we clearly addressed to the case where the element in regular. 
3. When considering Drazin invertibility of a ring element, a usefull reasoning is by considering powers. The elements of the form $t+1-t t^{-}$have powers with a special structure, as in Lemma 2.6:

Given a regular $t \in R$ and a natural $k$,

$$
\left(t+1-t t^{-}\right)^{k+1}=t\left(t+1-t t^{-}\right)^{k}+1-t t^{-} .
$$

The proof is done by induction. Simple calculations show the result holds for $k=1$.

Note that $\left(t+1-t t^{-}\right)^{k+1}=\left(t+1-t t^{-}\right)^{k}\left(t+1-t t^{-}\right)$which equals, by the induction step,

$$
\left(t\left(t+1-t t^{-}\right)^{k-1}+1-t t^{-}\right)\left(t+1-t t^{-}\right) .
$$

We obtain $t\left(t+1-t t^{-}\right)^{k}+1-t t^{-}$.

4. The invertibility of $a^{2} a^{-}+1-a a^{-}, a^{-} a^{2}+1-a^{-} a, a+1-a a^{-}, a+1-a^{-} a$ is independent of the choice of $a^{-}$. What can be said when considering the units in Proposition 2.2 and in Proposition 2.3?

5. We have shown that $i(a)=k+1$ if and only $i\left(a^{2} a^{-}+1-a a^{-}\right)=k$, for $k \geq 1$. We have also proved $i(A)=k+1$ if and only $i\left(A+1-A A^{-}\right)=k$, for $k \geq 1$, if $A$ is a square matrix over an algebraically closed field. Is the result also valid for, say, regular rings?

6. A positive answer for the previous item would provide the equivalence between $i\left(a^{2} a^{-}+\right.$ $\left.1-a a^{-}\right)=k$ and $i\left(a+1-a a^{-}\right)=k$, and in this case it is independent of the choice of $a^{-}$.

7. The previous question is part of a more vast and structural one: does $i(1-x y)=k$ imply $i(1-y x)=k$ ? When $k=0$ it is a well known result.

\section{Acknowledgment}

The authors wish to thank Professor C. Schmoeger for kindly having provided overprints of his papers.

\section{References}

[1] N. Castro González, Additive perturbation results for the Drazin inverse, Linear Algebra Appl., 397 (2005), 279-297.

[2] R.E. Cline, An application of representation of a matrix, MRC Technical Report, 592, 1965.

[3] M.P. Drazin, Pseudo inverses in associative rings and semigroups, Amer. Math. Monthly, 65 (1958), 506-514.

[4] R.E. Hartwig, J. Luh, On finite regular rings, Pacific J. Math., 69 (1977), no. 1, 73-95. 
[5] R.E. Hartwig, P. Patricio, A note on power bounded matrices, submitted.

[6] R.E. Hartwig, P. Patricio, R. Puystjens, Diagonalizing triangular matrices via orthogonal Pierce decompositions, Linear Algebra Appl., 401 (2005), 381-391.

[7] R.E. Hartwig, J. Shoaf, Group Inverses and Drazin inverse of bidiagonal and triangular Toeplitz matrices, J. Austral. Math. Soc. Ser. A, 24 (1977), no. 1, 10-34.

[8] R.E. Hartwig, G. Wang and Y. Wei, Some additive results on Drazin inverses, Linear Algebra Appl., 322 (2001), no. 1-3, 207-217.

[9] P. Patricio, R. Puystjens, Generalized invertibility in two semigroups of a ring, Linear Algebra Appl., 377 (2004), 125-139.

[10] R. Puystjens, R.E. Hartwig, The group inverse of a companion matrix. Linear and Multilinear Algebra, 43 (1997), no. 1-3, 137-150.

[11] S. Roman, Advanced linear algebra, GTM, Springer-Verlag, 2005.

[12] C. Schmoeger, On a class of generalized Fredholm operators. I. Demonstratio Math., 30 (1997), no. 4, 829-842.

[13] C. Schmoeger, On Fredholm properties of operator products. Math. Proc. R. Ir. Acad. 103 A (2003), no. 2, 203-208. 